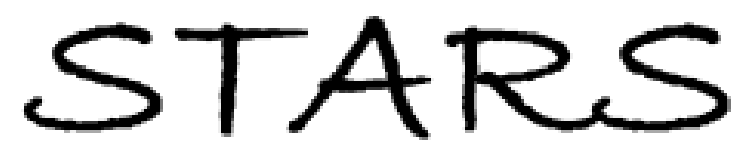

University of Central Florida

STARS

$1-1-2002$

\title{
Induced modulation instability of partially spatially incoherent light with varying perturbation periods
}

\author{
Zhigang Chen \\ Jill Klinger \\ Demetrios N. Christodoulides \\ University of Central Florida
}

Find similar works at: https://stars.library.ucf.edu/facultybib2000

University of Central Florida Libraries http://library.ucf.edu

This Article is brought to you for free and open access by the Faculty Bibliography at STARS. It has been accepted for inclusion in Faculty Bibliography 2000 s by an authorized administrator of STARS. For more information, please contact STARS@ucf.edu.

\section{Recommended Citation}

Chen, Zhigang; Klinger, Jill; and Christodoulides, Demetrios N., "Induced modulation instability of partially spatially incoherent light with varying perturbation periods" (2002). Faculty Bibliography 2000s. 3120. https://stars.library.ucf.edu/facultybib2000/3120

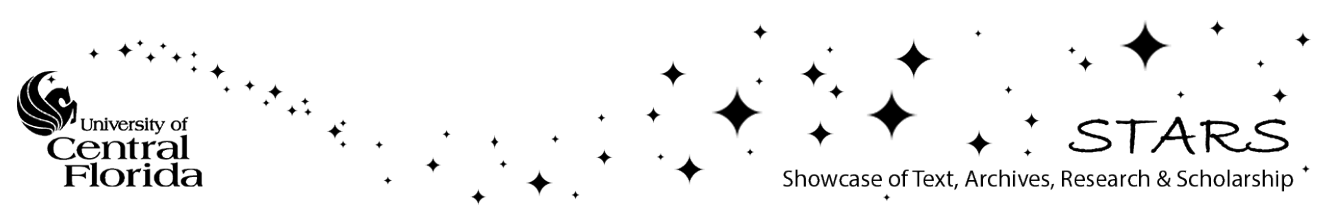




\title{
Induced modulation instability of partially spatially incoherent light with varying perturbation periods
}

\author{
Zhigang Chen* and Jill Klinger \\ Department of Physics and Astronomy, San Francisco State University, San Francisco, California 94132 \\ Demetrios N. Christodoulides \\ School of Optics/CREOL, University of Central Florida, Orlando, Florida 32816
}

(Received 4 June 2002; revised manuscript received 26 August 2002; published 3 December 2002)

\begin{abstract}
We demonstrate experimentally that a periodic perturbation on a partially spatially incoherent optical beam induces modulation instability that depends strongly on the perturbation periods as well as on the strength of the nonlinearity and the degree of spatial coherence. At a fixed value of the nonlinearity and coherence, the incoherent modulation instability has a maximum growth at a preferred perturbation period (or spatial frequency), leading to the formation of ordered patterns. While the nonlinearity in our photorefractive system is inherently anisotropic, pattern control and pattern switching with anisotropic coherence is readily realized. Our experimental observations are in good agreement with theoretical predictions.
\end{abstract}

DOI: 10.1103/PhysRevE.66.066601

PACS number(s): 42.65.Tg

Modulation instability (MI) is a universal phenomenon that exists in many nonlinear systems. In optics, small perturbations in amplitude or phase of optical waves tend to grow exponentially as a result of MI, manifested by the interplay of nonlinearity and diffraction/dispersion $[1,2]$. MI is of interest because it is a crucial issue to be considered in soliton formation and instabilities, in both time and space [2]. In fact, MI typically occurs in the same parameter region where solitons are observed, and thus it is considered as a precursor of solitons. In the spatial domain, because of MI, a broad optical beam disintegrates into many filaments during propagation in a self-focusing nonlinear medium [1]. Often the filaments arising from the breakup form structures akin to trains of optical spatial solitons [3]. For a narrow soliton beam, self-focusing exactly balances diffraction, whereas for a broad beam fragmentation occurs due to noise and nonlinearity as there is no such balance for the whole beam. Thus, as in many configurations in nonlinear optics, the same focusing nonlinearity leads to transverse instability and optical pattern formation.

Although coherent MI (MI of a coherent wave) is by now well known and understood, incoherent MI (MI of a partially coherent wave or a weakly correlated wave front) was brought into attention only recently [4], following the first demonstration of incoherent optical solitons [5,6]. Incoherent MI was predicted to occur in noninstantaneous self-focusing nonlinear media, provided that the nonlinearity exceeds a certain threshold condition. To elucidate this threshold condition, let us consider a partially incoherent source with a Lorentzian-like angular power spectrum. In this case, following the analysis of Ref. [4], the growth rate $g(\alpha)$ of MI can be found as

$$
\frac{g(\alpha)}{k}=-\left(k_{x 0} / k\right)(|\alpha| / k)+(|\alpha| / k) \sqrt{\frac{\kappa I_{0}}{n_{0}}-\left(\frac{\alpha}{2 k}\right)^{2}},
$$

\footnotetext{
*FAX: (415) 338-2178. Email address: zchen@stars.sfsu.edu
}

where $k$ is the wave vector, $\alpha$ is the spatial wave vector (or $2 \pi$ times the spatial frequency of the MI perturbations), $I_{0}$ is the uniform background intensity, $n_{0}$ is the linear refractive index of the material, and $\kappa=d(\delta n) / d I$ is the marginal nonlinear index change evaluated at $I_{0}$. In addition, $\theta_{0}=k_{x 0} / k$ in Eq. (1) represents the width of the angular power spectrum, which in turn describes the degree of spatial coherence of the beam. A smaller $\theta_{0}$ corresponds to higher coherence and in the limit of $\theta_{0}=0$, the beam becomes fully coherent. From Eq. (1), it is easy to see that, for a given degree of coherence, incoherent MI occurs only when the quantity $\kappa I_{0}$ exceeds a specific threshold, that is,

$$
\kappa I_{0} / n_{0}>\theta_{0}^{2},
$$

where $\kappa I_{0}=\Delta n_{\mathrm{NL}}$ represents the nonlinear index (NL) change. For a saturable nonlinearity as in photorefractive materials, this nonlinear index change is controlled conveniently by a bias field along with the intensity of the beam [3]. Equations (1) and (2) indicate clearly that incoherent MI has two unique features. The first is that the growth rate of incoherent MI is a function of the spatial frequency $\alpha$. Only for certain spatial frequencies, the MI has a maximum growth. The second is that, unlike coherent MI, incoherent MI occurs only when the value of the nonlinearity exceeds a threshold imposed by the degree of spatial coherence. The more incoherent a source is, the higher the nonlinearity (or the nonlinear index change) is needed for incoherent MI to occur. Below this threshold, incoherent MI is entirely eliminated. On the other hand, in the coherent limit $\left(\theta_{0}=0\right)$, there is no such threshold. These predictions from the theory of one-dimensional (1D) (i.e., one transverse dimension) incoherent MI [4], were soon followed by a series of experimental demonstrations that are unique to incoherent waves and that cannot be realized in the coherent regime. These include, for example, incoherent antidark solitons [7], incoherent pattern formation $[8,9]$, and soliton clustering in weakly correlated wave fronts [10]. Very recently, a comprehensive study of 1D incoherent MI was conducted, providing good agree- 


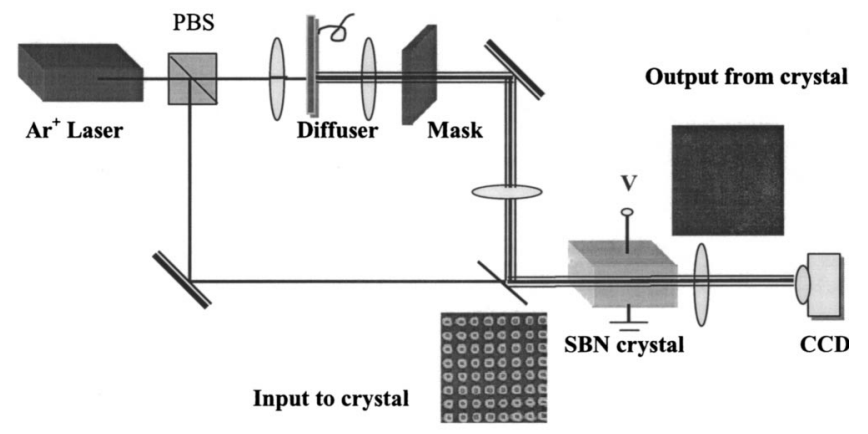

FIG. 1. Experimental setup.

ment between theory and experiments [11]. Meanwhile, the theory of 2D (i.e., two transverse dimensions) incoherent MI was also developed [12], which predicted novel pattern formation via symmetry breaking in nonlinear partially coherent wave fronts.

In this paper, we report on the first direct observation of modulation instability of a partially spatially incoherent beam as a function of the perturbation period. We provide experimental evidence that such induced incoherent MI depends strongly on the perturbation period (or spatial frequency), apart from its dependence on the coherence of the beam and the strength of the nonlinearity. In a way very different from previous experiments on incoherent MI, which was driven by intrinsic noise (such as defects and striations in the crystal) $[8,10]$ or seeding noise from cross-phasemodulation [9], here the incoherent beam is spatially modulated directly by amplitude masks with various modulation periods. By actively seeding spatial noise onto an otherwise uniform incoherent beam, we demonstrate that the induced modulation instability has a maximum growth at a certain preferential perturbation period, leading to formation of ordered patterns. At a fixed spatial frequency of perturbation, the strength of the nonlinearity and the degree of spatial coherence of the beam control the growth of modulation instability and pattern formation. We also find that by introducing anisotropic coherence in the spatially modulated beam, pattern formation and pattern switching can be realized. To our knowledge, this is the first observation of pattern formation via induced symmetry breaking in nonlinear weakly correlated systems.

In our experiments, a partially spatially incoherent beam is generated by converting an argon ion laser beam ( $\lambda$ $=488 \mathrm{~nm}$ ) into a quasimonochromatic light source with a rotating diffuser (Fig. 1). The spatial coherence of the beam is varied by changing the relative position of the diffuser and is monitored from the speckle size when the diffuser is set to rest. The speckle size is roughly equal to the spatial coherence length $l_{c}$, within which any two points on the wave front remain phase correlated. A biased photorefractive crystal (strontium barium niobate, SBN:61; $5 \times 5 \times 20 \mathrm{~mm}^{3}$ ) is used as the medium that provides noninstantaneous nonlinearity, as the rotating diffuser creates random phase fluctuations on a time scale much faster than the response time of the crystal. This noninstantaneous nature of the photorefractive nonlinearity is essential for incoherent solitons and MI to occur $[4-11]$. The incoherent beam is extraordinarily po- larized, and is spatially modulated by passing it through an amplitude mask that creates a gridlike intensity pattern at the crystal input face. Such a periodic spatial modulation, as previously generated from multibeam interference [9], provides "seeding noise" for observation of induced modulation instability. In addition to the incoherent beam, a broad and uniform ordinarily polarized coherent beam from the same laser is used as "dark illumination" to fine tune the nonlinearity [3]. In the linear region (no bias field), the intensity grids diffract dramatically as expected from incoherent light, and as the whole beam propagates through the 20-mm-long crystal, diffraction washes out the fine structures in the beam, leaving a nearly uniform intensity pattern at the crystal output (Fig. 1). Since the amplitude masks are used for seeding spatial modulation at input of an otherwise uniform beam, which gives a high-contrast periodic intensity pattern, we do not have control for the strength of the seeding noise, but rather the period of perturbation. Our motivation is to study how the beam breaks up in the nonlinear regime, and how the breakup depends on the perturbation period as well as on the degree of spatial coherence and the strength of the nonlinearity. We emphasize that, for the nonlinear region where we are to focus on, the incoherent MI is induced by the seeding spatial modulation. In other words, our experimental conditions are so chosen that, should the amplitude mask be removed so to provide a uniform input beam, the beam would remain fairly uniform at output even with the nonlinearity on. Of course, if the nonlinearity/coherence is increased further, the beam can break up by itself due to the development of incoherent MI from noise inherent in the system, as studied previously $[8,11]$.

Typical experimental results are presented in Fig. 2, where the intensity patterns from the output of the biased crystal are displayed as a function of perturbation periods. In these experiments, the spatial coherence length of the beam is fixed at about $15 \mu \mathrm{m}$, as estimated from the average speckle size when the diffuser is set to rest, and the bias field is fixed at $1500 \mathrm{~V} / \mathrm{cm}$. Each time after we change an amplitude mask so as to vary the perturbation period, the intensity of the partially incoherent beam is reset to the same level so that the ratio between the intensities of the modulated incoherent beam and that of the uniform background beam remains at a constant value (about 3). At these experimental conditions, we find that the growth of incoherent MI is not appreciable if we remove the amplitude mask, as the beam remains uniform after nonlinear propagation through the crystal. However, new features are observed if we insert the amplitude mask with appropriate modulation periods. In Fig. 2 , the left column shows the intensity distribution in real space, while the right column shows the corresponding spatial power spectrum. From top to bottom, the perturbation period is increased gradually. Clearly, the incoherent beam remains fairly uniform when the perturbation period is too small (or the spatial frequency of perturbation is too high). As the period is increased to $65 \mu \mathrm{m}$, the intensity pattern starts to break up into 1D stripes, and this breakup becomes more pronounced at $85 \mu \mathrm{m}$. Meanwhile, the amplitude of the spatial power spectrum increases in the Fourier space, indicating that the growth of induced modulation instability at 


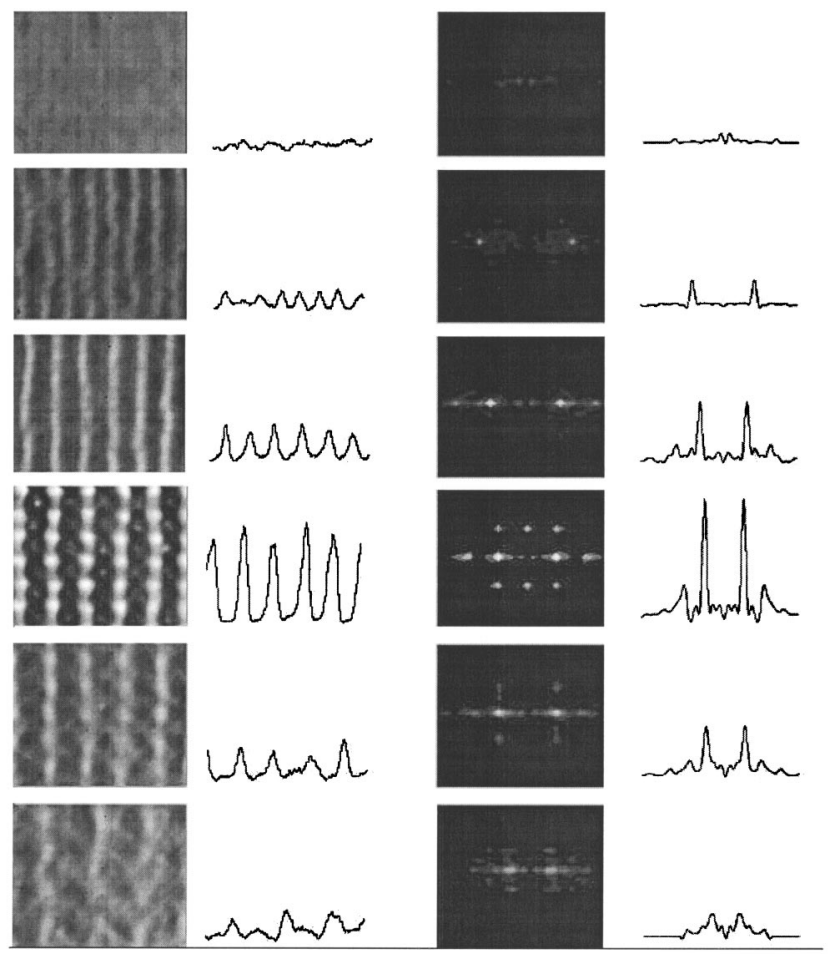

FIG. 2. Induced MI of a partially incoherent beam with varying spatial perturbation periods. Shown are photographs of intensity patterns taken at crystal output (left), along with their corresponding spatial power spectrum (right). All data were taken under the same experimental conditions (coherence: $15 \mu \mathrm{m}$; bias field: $1500 \mathrm{~V} / \mathrm{cm}$ ), except that the period of perturbation was varied. From top to bottom, the periods of perturbation are 40, 65, 85, 100, 110, and 150 $\mu \mathrm{m}$.

these spatial frequencies becomes stronger. When the perturbation period is set to $100 \mu \mathrm{m}$, the 1D stripes break up further into 2D filaments, as can been seen also from the corresponding power spectrum where the spatial frequency has vertical as well as horizontal component. In fact, the amplitude of power spectrum reaches a maximum, indicating the growth rate of MI has a peak at this spatial frequency. As the perturbation period is further increased, the intensity structures of 1D breakup reappear, and eventually the beam becomes fairly uniform (MI disappears). This scanning of perturbation period clearly shows that the gain of induced incoherent MI indeed depends on spatial frequency, as predicted by theory [4]. This can be seen even more clearly from Fig. 3, where both the modulated peak intensity and the amplitude of the power spectrum are plotted as a function of the spatial frequency. For the experiments of Figs. 2 and 3, the maximum growth of incoherent MI occurs at a spatial frequency of $0.063 / \mu \mathrm{m}$. In addition, we have performed a series of experiments of varying perturbation periods for induced MI under different conditions. Figure 4 shows anther set of data obtained at a slightly different spatial coherence $\left(l_{c}=18 \mu \mathrm{m}\right)$, where the same sequence of pattern formation is observed. It can be seen that, under these experimental conditions, the incoherent MI has a maximum gain around a perturbation period of $110 \mu \mathrm{m}$, which corresponds to a spatial frequency of $0.057 / \mu \mathrm{m}$. From these experimental results, it seems that, the maximum growth of MI, which is characterized by a strong 2D breakup, shifts to a lower spatial frequency as the coherence increases. This feature is not expected from the 1D theory of incoherent MI, as has been addressed recently in the study of $1 \mathrm{D}$ incoherent MI without seeding noise [11]. According to the predictions from 1D theory [4], the spatial frequency for a maximum MI gain should increase monotonically as the coherence increases at a fixed value of nonlinearity, and also increase monotonically as the strength of the nonlinearity increases at a fixed value of coherence. These predictions have not been observed clearly in experiments. It seems that a 2D theory of incoherent MI that takes into account the anisotropic nonlinearity is needed to explain some of the experimental observations.

Having showed how the induced MI depends on the spatial frequency of modulation, we study next the effect of nonlinearity and coherence on incoherent MI at a fixed perturbation period. We increase the strength of the nonlinearity by increasing the voltage applied across the 5-mm-wide crystal, while keeping the intensity of the beam unchanged. Figure 5(a) shows the photographs of intensity patterns taken at output of crystal as the voltage is increased gradually. The perturbation period is fixed at $110 \mu \mathrm{m}$, and the spatial coherence of the beam at $25 \mu \mathrm{m}$. The incoherent beam remains fairly uniform at $500 \mathrm{~V}$, but breaks up into $1 \mathrm{D}$, and then $2 \mathrm{D}$, structures at higher voltages. Similar sequence of pattern formation due to incoherent MI is observed for varying coherence as shown in Fig. 5(b), for which the perturbation period is fixed at $75 \mu \mathrm{m}$, and the bias field at $1520 \mathrm{~V} / \mathrm{cm}$. When the beam is made too incoherent, it remains uniform as the threshold for incoherent MI has not been reached for the nonlinearity provided. As the coherence of the beam is increased gradually, the nonlinearity reaches the threshold for $1 \mathrm{D}$ and then 2D incoherent MI, leading to patterns of ordered stripes and 2D quasisoliton filaments. These observations are in agreement with theoretical predictions, and are consistent with previous experiments of incoherent MI mediated either by preferential noise along the direction of crystal striations $[8,10,11]$ or by noise seeded through cross-phase modulation [9]. We point out that our seeding noise is not anisotropic, and thus it does not provide a preferential direction for MI to growth. In addition, the striation lines in our SBN crystal are located more diagonally than horizontally/vertically. Yet, the stripes from 1D breakup always start to orient in the vertical direction (Fig. 5) because of anisotropic photorefractive nonlinearity involved.

It is now clear that both theoretical and experimental studies have revealed the two main features of incoherent MI: the presence of a well-defined threshold that depends on the nonlinearity and coherence, and the presence of a maximum growth rate that depends on the spatial frequency. The intuitive picture for the first one is quite straightforward. For a partially coherent wave front, the periodic perturbation on top of it tends to grow because of the self-focusing nonlinearity, but meanwhile, the perturbation also tends to wash out during propagation due to the incoherence. Thus, below the threshold, any small perturbations on the incoherent beam cannot be amplified because the diffusive washout effect is stronger than the nonlinear self-focusing effect. Only above 
(a)

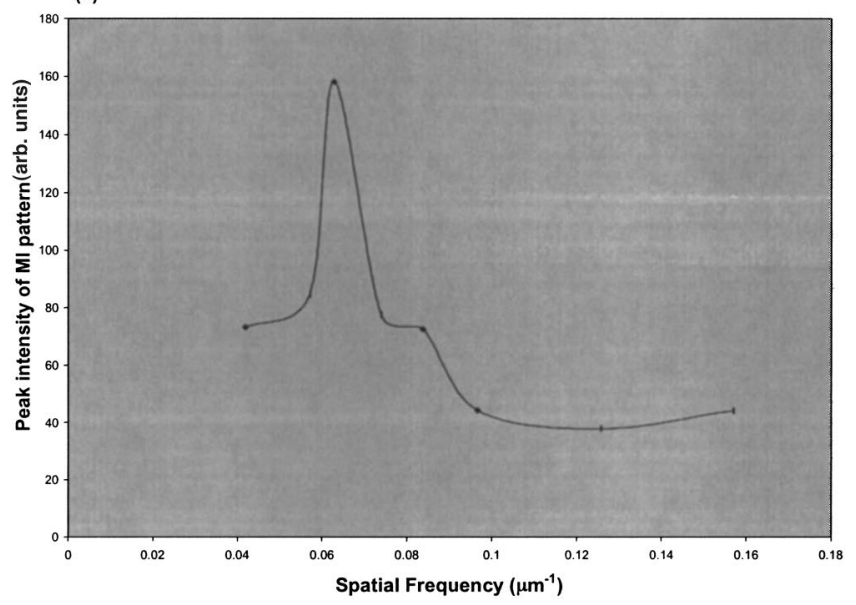

(b)

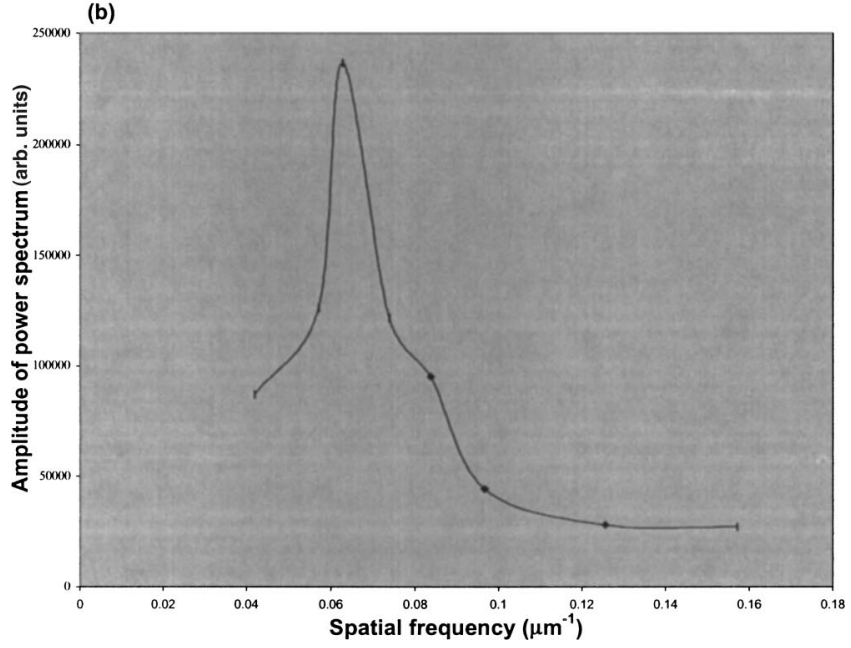

FIG. 3. Plot of the peak intensity of induced MI (a) and the amplitude of power spectrum (b) from Fig. 2 as a function of the spatial frequency.

the threshold can the perturbation grow, thus leading to incoherent MI. This is in contradistinction with coherent MI, which has no threshold, as explained earlier [11]. The intuitive picture for the second one is as follows. Once above the threshold for incoherent MI, the filaments from the breakup of the beam tend to form solitons. But for a given value of nonlinearity and coherence, solitons cannot form at any size, as there is a unique existence condition for soliton formation [3]. In fact, the size of the filaments which is most close to the condition for soliton formation determines the modulation scale or the spatial frequency that will enjoy the maximum MI growth.

Finally, for the 2D incoherent MI, it is interesting to explore the role played by symmetry breaking in MI and pattern formation, as proposed in a recent theoretical paper [12]. Specifically, anisotropy between two transverse dimensions such as that arising from nonlinearity, coherence (correlation statistics), or noise may be introduced in a nonlinear system to study MI and the associated nonlinear dynamics. Such anisotropy leads to symmetry breaking and to pattern formation in nonlinear partially coherent wave fronts. As mentioned before, in our photorefractive SBN crystal, the non- (a)

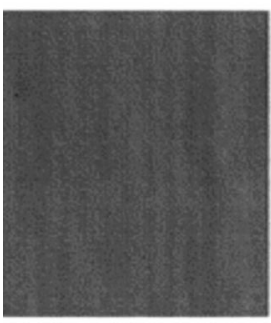

(d)

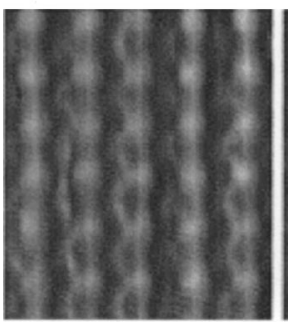

(b)

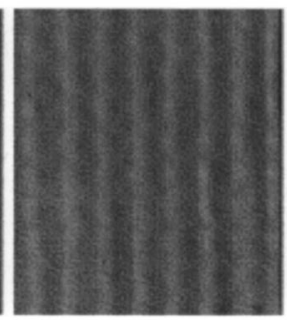

(e)

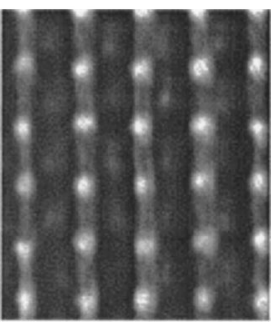

(c)

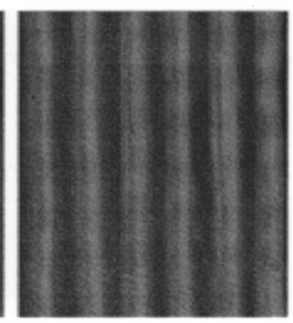

(f)

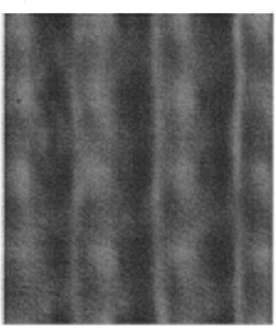

FIG. 4. Another set of data showing pattern formation at various perturbation periods. From (a) to (f), the periods of perturbation are $40,65,85,100,110$, and $150 \mu \mathrm{m}$.

linearity is inherently anisotropic because of the anisotropic nature of the electro-optic coefficients, which gives rise to a highly anisotropic nonlinear response (see the last two references in Ref. [1]). When a dc field is applied along the horizontal $x$ direction, a broad extraordinarily polarized beam always experiences a higher refractive index change in $x$ direction than in the vertical $y$ direction. This is why an incoherent beam always breaks up first in the $x$ direction, forming vertical stripes (Figs. 2-5). While we cannot change the anisotropy of the nonlinearity inherent in our photorefractive system, it is possible to change the anisotropy in the spatial coherence of the beam. In other words, we can simply make the spatial correlation length different in the two transverse directions. This anisotropic spatial coherence has already

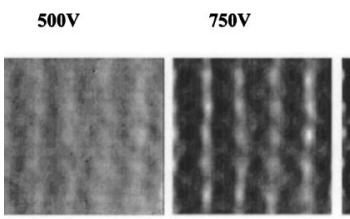

$1000 \mathrm{~V}$

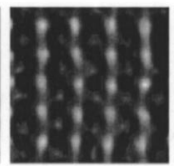

$1500 \mathrm{~V}$

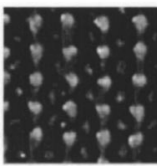

2000V

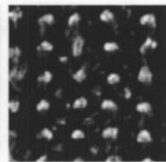

$5 \mu \mathrm{m}$

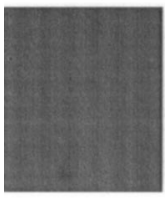

$10 \mu \mathrm{m}$

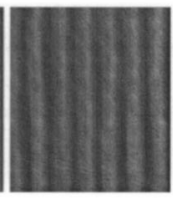

$18 \mu \mathrm{m}$

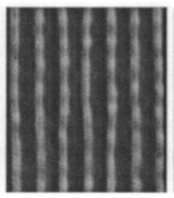

$24 \mu \mathrm{m}$

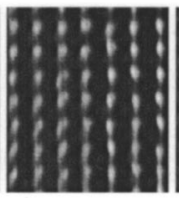
$50 \mu \mathrm{m}$

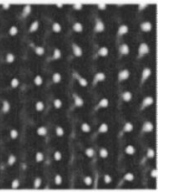

FIG. 5. Induced MI of a partially incoherent beam at a fixed spatial perturbation period. Top: Varying bias field at a fixed coherence of $25 \mu \mathrm{m}$ and a fixed perturbation period of $110 \mu \mathrm{m}$, Bottom: Varying coherence at a fixed bias field of $1520 \mathrm{~V} / \mathrm{cm}$ and a fixed perturbation period of $75 \mu \mathrm{m}$. 


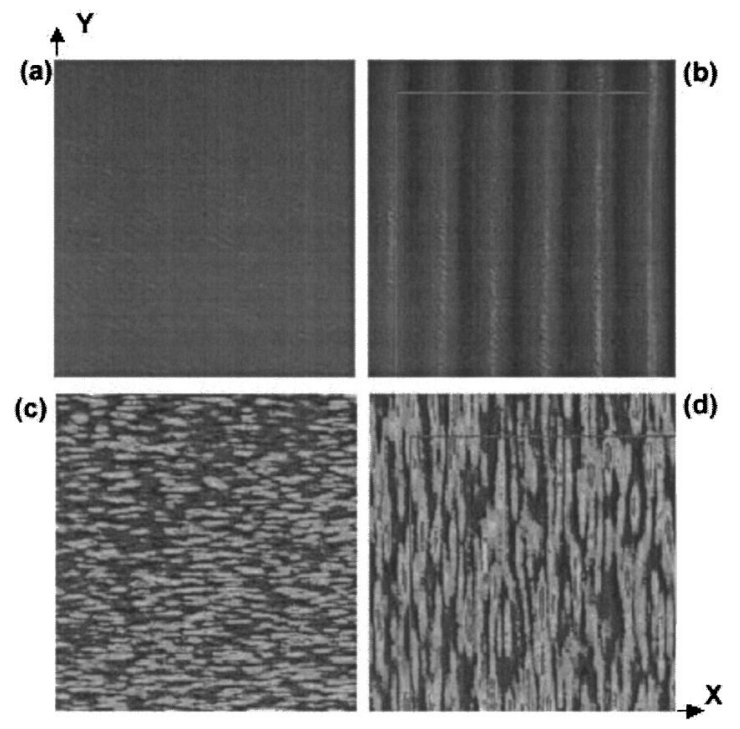

FIG. 6. Pattern formation and switching via symmetry breaking in spatial coherence. (a) and (b): photographs of intensity patterns taken at crystal output after nonlinear propagation. (c) and (d): speckled patterns showing anisotropic coherence.

been used to generate elliptical spatial solitons [13] and to eliminate the transverse modulation instability of onedimensional solitons in a Kerr-like bulk medium [14]. Figure 6 shows a typical example. We start with isotropic coherence (with circular speckles obtained by using a circular lens), and we have the coherence and the nonlinearity set to a value that is below the threshold for incoherent MI, so the beam remains uniform at the output of crystal even with the nonlinearity on [Fig. 6(a)]. We then make the beam more coherent in the $x$ direction [with elliptical speckles obtained by using a cylindrical lens as shown in Fig. 6(c)] and keep all the other experimental conditions unchanged. The otherwise uniform pattern in the nonlinear region now turns into a stripe pattern [Fig. 6(b)]. This is because the increase of coherence in $x$ direction enables incoherent MI to occur, which breaks up the beam first in the $x$ direction. On the other hand, when a stripe pattern is obtained initially under isotropic coherence, by making the beam more coherent in $y$ direction and less coherent in $x$ direction (Fig. 6(d)), we observe the inverse transition from a stripe pattern to a uniform pattern. In principle, such transition can happen between a stripe pattern and a two-dimensional gridlike pattern, and such pattern formation can be realized using anisotropic seeding noise as well as anisotropic spatial correlation function [12]. This will be the subject of future study. Our preliminary results of Fig. 6 suggest that pattern formation and switching with incoherent light can be a direct outcome of symmetry breaking in the spatial coherence.

In summary, we have demonstrated nonlinear propagation and modulation instability of a partially spatially incoherent beam driven by a seeding perturbation and the noninstantaneous self-focusing nonlinearity in the photorefractive medium. Main features of incoherent MI and novel pattern formation via symmetry breaking are observed in our experiments. Since nonlinear systems involving partial coherence, weak correlation, or symmetry breaking are abundant in nature, our results may prove relevant to other fields of nonlinear physics.

This research was supported by the Research Corporation and funding from the U.S. Army Research Office. We acknowledge the assistance from M. Segev and G. Salamo, and useful discussions with S. Li, T. Carmon, and C. Denz.
[1] E. M. Dianov et al., Opt. Lett. 14, 1008 (1989); P. V. Mamyshev et al., J. Opt. Soc. Am. B 11, 1254 (1994); M. D. IturbeCastillo et al., Opt. Lett. 20, 1853 (1995); M. I. Carvalho et al., Opt. Commun. 126, 167 (1996); A. V. Mamaev et al., Europhys. Lett. 35, 25 (1996); Phys. Rev. A 54, 870 (1996).

[2] Y. S. Kivshar and D. E. Pelinovsky, Phys. Rep. 331, 117 (2000); G. P. Agrawal, Nonlinear Fiber Optics (Academic, San Diego, CA, 1995).

[3] M. Segev and G. I. Stegeman, Phys. Today 51(8), 42 (1998); G. I. Stegeman and M. Segev, Science 286, 1518 (1999).

[4] M. Soljacic, M. Segev, T. Coskun, D. N. Christodoulides, and A. Vishwanath, Phys. Rev. Lett. 84, 467 (2000).

[5] M. Mitchel, Z. Chen, M. Shih, and M. Segev, Phys. Rev. Lett. 77, 490 (1996); M. Mitchell and M. Segev, Nature (London) 387, 880 (1997).

[6] Z. Chen, M. Mitchell, M. Segev, T. Coskun, and D. N. Christodoulides, Science 280, 889 (1998).
[7] T. H. Coskun, D. N. Christodoulides, Y. Kim, Z. Chen, and M. Segev, Phys. Rev. Lett. 84, 2374 (2000).

[8] D. Kip, M. Soljacic, M. Segev, E. Eugenieva, and D. N. Christodoulides, Science 290, 495 (2000).

[9] J. Klinger, H. Martin, and Z. Chen, Opt. Lett. 26, 271 (2001).

[10] Z. Chen, S. Sears, H. Martin, M. Segev, and D. N. Christodoulides, Proc. Natl. Acad. Sci. U.S.A. 99, 5523 (2002).

[11] D. Kip, M. Soljacic, M. Segev, S. M. Sears, and D. N. Christodoulides, J. Opt. Soc. Am. B 19, 502 (2002).

[12] S. M. Sears, M. Soljacic, D. N. Christodoulides, and M. Segev, Phys. Rev. E 65, 036620 (2002).

[13] E. D. Eugenieva, D. N. Christodoulides, and M. Segev, Opt. Lett. 25, 972 (2000).

[14] C. Anastassiou, M. Soljacic, M. Segev, D. Kip, E. D. Eugenieva, D. N. Christodoulides, and Z. Musslimani, Phys. Rev. Lett. 85, 4888 (2000). 